\title{
Classification Photometry for Ten dM Planet Hosts
}

\author{
Robert F. Wing \\ Astronomy Department, Ohio State University \\ 140 West 18th Avenue, Columbus, Ohio 43210, USA \\ email: wing@astronomy . ohio-state.edu
}

\begin{abstract}
Spectral classifications and color temperatures based on narrow-band $\mathrm{TiO} / \mathrm{CN}$ photometry are given for ten $\mathrm{dM}$ stars that are known to be planet hosts.
\end{abstract}

Keywords. stars: late-type, stars: classification, techniques: photometric

\section{Introduction}

As the search for extra-solar planets continues, lower main-sequence stars of spectral type $M$ have proved to be a fertile hunting ground, albeit with a lower detection rate than that of solar-type stars. Owing to their small masses, $\mathrm{M}$ dwarfs are relatively easily moved by orbiting companions, and the immediate solar neighborhood contains numerous $\mathrm{M}$ dwarfs that are comfortably bright for accurate radial-velocity work with large telescopes.

In an effort to improve the basic data for the particular $\mathrm{M}$ dwarfs that have been found to host planets, I have been observing them on my system of narrow-band $\mathrm{TiO} / \mathrm{CN}$ classification photometry. The data yield precise spectral classifications tied to the MK system and color temperatures that can be calibrated in terms of effective temperature.

\section{The photometric system}

My 8-color narrow-band photometric system, originally defined for use with photomultipliers, measures the strongest bands of $\mathrm{TiO}, \mathrm{VO}$, and $\mathrm{CN}$, as well as clean continuum points, in the 7000-11000 $\AA$ spectral region. It was designed for the study of M-type stars of all luminosities; one of its original objectives was to obtain internally consistent spectral classifications for $\mathrm{M}$ dwarfs on the same TiO-based scale as for giants and supergiants (Wing 1973). Recently the system has been used to identify a set of standard stars that define the mean solar-neighborhood main sequence from type K4 V to M6 V (Wing \& van der Bliek 2009). Details of the photometric system and its calibrations are given in MacConnell, Wing, \& Costa (1992) and in Wing (2011).

Currently observations are obtained with a CCD and a set of six large-format filters that are nearly identical to the first six filters of the original 8-color system. A set of new (fainter) standard stars has been developed for use with the CCD.

\section{Observations}

The observations discussed here were obtained at the CTIO/SMARTS 0.9-m telescope in Chile. Ten dM stars known to have planets have been observed to date and are listed in Table 1 with their common, GJ, and LHS names. These do not represent a complete list of known dM planetary hosts; rather, they are the ones that were known to me, and accessble from Chile, at the times of my observing runs. 

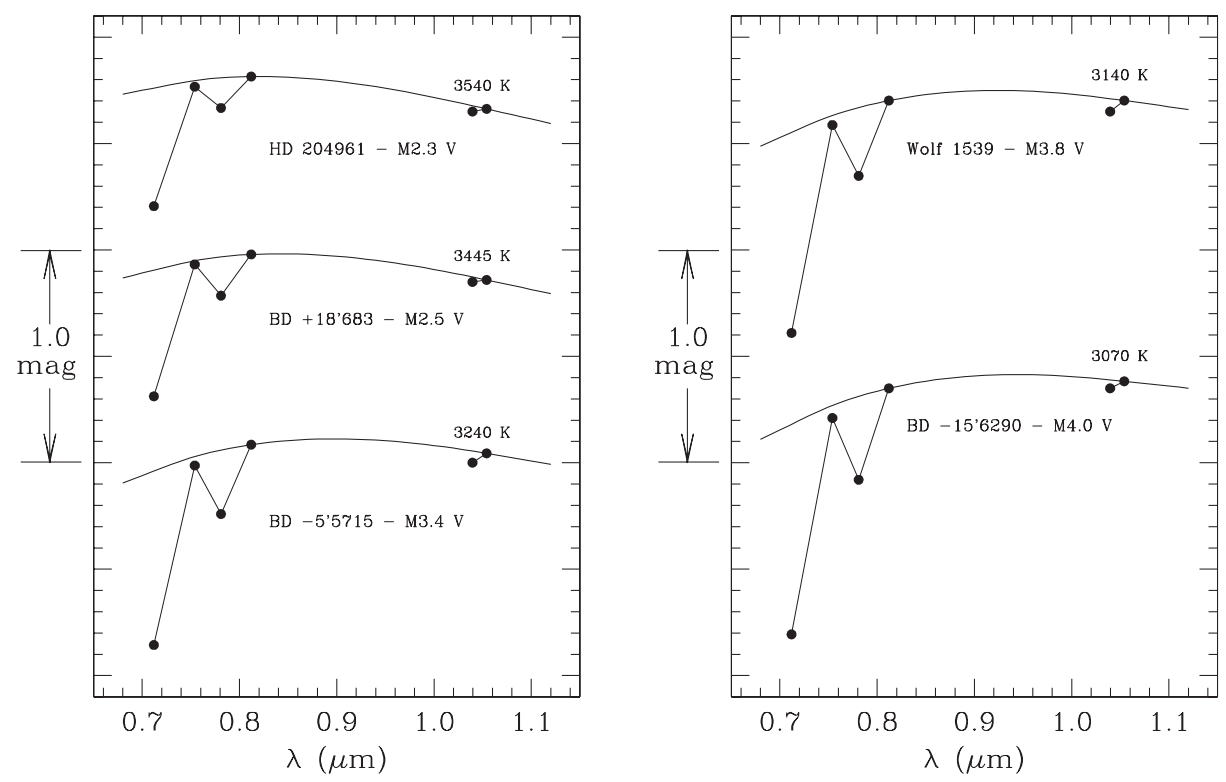

Figure 1. Six-color photometry, reduced to an absolute flux system and plotted on a magnitude scale with an arbitrary zero point for each star, is shown for $5 \mathrm{dM}$ stars known to be planetary hosts. The color temperature of the fitted BB continuum is shown above each spectrum. The spectral types are from the depression due to $\mathrm{TiO}$ in the first filter, relative to the continuum.

Table 1. Results from 6-color photometry.

\begin{tabular}{lcc|rlc}
\hline Star & GJ & LHS & $I(104)$ & $T_{\text {color }}$ & Spectral Type \\
\hline HD 204961 & 832 & 3685 & 5.72 & $3540 \mathrm{~K}$ & M2.3 V \\
BD +18 683 & 176 & 196 & 6.94 & 3445 & M2.5 V \\
BD -5 5715 & 849 & 517 & 6.97 & 3240 & M3.4 V \\
BD -7 4003 & 581 & 394 & 7.18 & 3410 & M3.4 V \\
BD -15 6290 & 876 & 530 & 6.40 & 3070 & M4.0 V \\
Ross 905 & 436 & 310 & 7.43 & 3310 & M3.0 V \\
Wolf 1539 & 179 & - & 8.35 & 3140 & M3.8 V \\
GJ 317 & 317 & 2037 & 8.44 & 3265 & M3.8 V \\
GJ 1214 & 1214 & 3275 & 10.30 & 2790 & M4.6 V \\
Hip 79431 & - & - & 8.04 & 3285 & M3.3 V \\
\hline
\end{tabular}

The planets of six of these stars - BD $-5^{\circ} 5715$, BD $-15^{\circ} 6290$, Ross 905 , Wolf 1539 , GJ 317, and Hipparcos 79431 — were discovered by the precise Doppler measurements of planet search programs at Keck Observatory (e.g. Howard et al. 2010). The family of planets belonging to $\mathrm{BD}-7^{\circ} 4003$ were discovered with the HARPS spectrograph at La Silla, starting with Bonfils et al. (2005). The planet of HD 204961 was found at the Anglo-Australian Telescope (Bailey et al. 2009) and that of $\mathrm{BD}+18^{\circ} 683$ at the HobbyEberly Telescope (Endl et al. 2008). The planet of GJ 1214 has been observed in a variety of ways including transit observations (Charbonneau et al. 2009).

\section{Results}

The six-color "spectra" of five of the target stars are shown in Figure 1. The photometry has been reduced to a system of absolute fluxes and is plotted on a magnitude scale. 


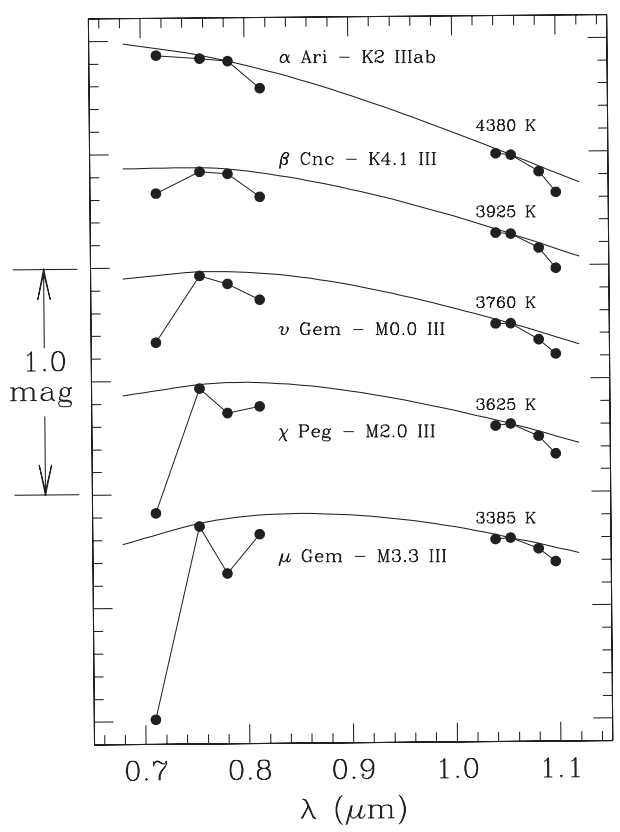

Figure 2. A sequence of giant stars, for comparison. Note that filter 4 is depressed in these stars - by CN, which is not present in the dwarfs. These bright stars were observed with an S-1 photomultiplier, the long-wavelength sensitivity of which allowed inclusion of two additional filters near 1.1 microns to measure the $(0,0)$ band of CN. From Wing (2011).

Blackbody curves fitted to the best continuum points (here filters 4 and 6) define a continuum with respect to which the depressions in other filters are measured.

Spectral types are based on a calibration of the depression at the first filter, which measures a strong band of $\mathrm{TiO}$. The $\mathrm{CN}$ molecule, which depresses filter 4 when present, is vanishingly weak in $\mathrm{M}$ dwarfs (see the spectra of giant stars shown in Figure 2).

The results are given in Table 1 . Here $I(104)$ is the flux in filter 5 at $10350 \AA$ expressed as a magnitude zeroed to the flux of Vega $(\alpha \mathrm{Lyr})$, and $T_{\text {color }}$ is from the blackbody fit.

\section{References}

Bailey, J., Butler, R.P., Tinney, C. G. et al. 2009, ApJ, 690, 743

Bonfils, X., Forveille, T., Delfosse, X. et al. 2005, A\& $A$, 443, L15

Charbonneau, D., Berta, Z. K., Irwin, J. et al. 2009, Nature, 462, 891

Endl, M., Cochran, W. D., Wittenmyer, R. A., \& Boss, A. P. 2008, ApJ, 673, 1165

Howard, A. W., Johnson, J. A., Marcy, G. W. et al. 2010, ApJ, 721, 1467

MacConnell, D. J., Wing, R.F., \& Costa, E. 1992, AJ, 104, 821

Wing, R.F. 1973, in IAU Coll. 50, Spectral Classification and Multicolour Photometry, ed. Ch. Fehrenbach \& B. E. Westerlund, Reidel, p. 209

Wing, R. F. 2011, in Astronomical Photometry - Past, Present, and Future, ed. E. F. Milone \& C. Sterken, Astrophysics and Space Science Library 373, Springer, p. 143

Wing, R.F. \& van der Bliek, N. S. 2009, in the 15th conference on Cool Stars, Stellar Systems, and the Sun, AIP Conf. Proc. 1094, 820 